\title{
Telemedicine for Glaucoma Patients in COVID-19 Era
}

\section{Hany Mahmoud}

Lecturer of Ophthalmology, Faculty of Medicine, Sohag University, Sohag, Egypt

Email:drhanymahmoud@gmail.com

In that Era with crowded hospitals and the need for social distance, telemedicine emerges as a solution for many problems. In ophthalmology Telemedicine has been started many years ago with limits. Now we have to widen the scope and applications of telemedicine (1-3)

Glaucoma patients needs a special follow-up regimen with intraocular pressure (IOP) measurement, fundus examination, and visual acuity (V/A) assessment. Regular visits to the hospital are required; however, in the telemedicine protocol, we can decrease their visits to the hospitals. $(4-6)$

Excluding emergencies, patients with glaucoma can follow the following protocol:

History taking: by phone or video call with health provider ${ }^{(3)}$

Visual acuity assessment: Patients can use Amsler's grid chart or smart applications for visual acuity and color vision.

Iop: I CARE tonometer can be a valuable tool; however, it is expensive. ${ }^{(7)}$

Visual field: There are Apps and devices to monitor the central 10, degrees including the commercially available preferential hyperacuity perimeter ForSee ${ }^{\circledR}$ device (Notal Vision) and discrimination apps such as $\mathrm{mVT}^{\circledR}$ (Vital Art and Science) application ${ }^{(8,9)}$. Smart phone retinal imaging is available, but not yet designed for patients to use at home. ${ }^{(10-13)}$

Video call: with the health care provider to discuss the condition and guide the patient to either the hospital or drug prescription. 
Future prospective: We aim at provide an optical coherence tomography (OCT) smart phone like apparatus. Intraocular devices for continuous IOP measurement are required.

Obstacles: Economic problems with some patients

Low-quality infrastructure in some places.

\section{Benefits:}

Decrease in hospital crowdness

Social distance in the era of the COVID-19.

\section{References:}

1. Get Your Clinic Ready for Coronavirus Disease 2019 (COVID-19) | CDC. https://www.cdc.gov/coronavirus/2019-ncov/hcp/clinic-preparedness.html. Accessed April 18, 2020.

2. Telemedicine during COVID-19: Benefits, limitations, burdens, adaptation | Healthcare IT News. https://www.healthcareitnews.com/news/telemedicine-duringcovid-19-benefits-limitations-burdens-adaptation. Accessed April 18, 2020.

3. Marsden J. An evaluation of the safety and effectiveness of telephone triage as a method of patient prioritization in an ophthalmic accident and emergency service. $\mathrm{J}$ Adv Nurs. 2000;31(2):401-409. [PubMed] [Google Scholar]

4. Chalk D., Smith M. Guidelines on glaucoma and the demand for services. Br J Heal Care Manag. 2013;19(10):476-481. [Google Scholar]

5. National Collaborating Centre for Acute Care. Glaucoma. Diagnosis and Managenement of Chronic Open Angle Glaucoma and Ocular Hypertension. Methods, Evidence \& Guidance. National Collaborating Centre for Acute Care (UK); 2009.

6. Chen E., Quérat L., Åkerstedt C. Self-tonometry as a complement in the investigation of glaucoma patients. Acta Ophthalmol. 2016;94(8):788792. [PubMed] [Google Scholar]

7. Tan S., Yu M., Baig N., Hansapinyo L., Tham C.C. Agreement of patient-measured intraocular pressure using rebound tonometry with Goldmann applanation tonometry (GAT) in glaucoma patients. Sci Rep. 2017;7:42067. [PMC free article] [PubMed] [Google Scholar]

8. Philippin H., Shah P., Burton M. Detecting possible glaucoma with only limited equipment: A crucial first step. Community Eye Heal J. 2012;25(79-80):4849. [Google Scholar] 
9. Lowry E.A., Hou J., Hennein L. Comparison of peristat online perimetry with the humphrey perimetry in a clinic-based setting. Transl Vis Sci Technol. 2016;5(4) [Google Scholar]

10. Vingrys A.J., Healey J.K., Liew S. Validation of a tablet as a tangent perimeter. Transl Vis Sci Technol. 2016;5(4) [Google Scholar]

11. Gan K, Liu Y, Stagg B, Rathi S, Pasquale LR, Damji K. Telemedicine for Glaucoma: Guidelines and Recommendations. Telemed e-Health. March 2020:tmj.2020.0009.

12. Lord R.K., Shah V.A., San Filippo A.N., Krishna R. Novel Uses of Smartphones in Ophthalmology. Ophthalmology. 2010;117(6) [Google Scholar]

13. Muiesan M.L., Salvetti M., Paini A. Ocular fundus photography with a smartphone device in acute hypertension. J Hypertens. 2017;35(8):16601665. [PubMed] [Google Scholar] 